\title{
Long-Term Follow-up in Patients with Spontaneous Intracerebral Hemorrhage Treated With or Without Surgical Intervention: a Large-Scale Retrospective Study
}

\author{
XianXiu Chen ${ }^{1,2} \cdot$ Yuan-Chih Su ${ }^{3,4} \cdot$ Chun-Chung Chen ${ }^{3,5,6}$. Jeng-Hung Guo ${ }^{5}$. Chih-ying Wu ${ }^{5,7}$. Sung-Tai Wei ${ }^{5}$. \\ Der-Cherng Chen ${ }^{5} \cdot$ Jung-Ju Lin $^{8} \cdot$ Shwn-Huey Shieh ${ }^{9} \cdot$ Cheng-Di Chiu $^{5,6,10}$
}

Published online: 20 February 2019

(C) The American Society for Experimental NeuroTherapeutics, Inc. 2019

\begin{abstract}
Debates regarding the most beneficial medical or surgical procedures for patients with spontaneous intracerebral hemorrhage ( $\mathrm{sICH})$ are still ongoing. We aimed to evaluate the risk of subsequent vascular disease and mortality in patients with sICH treated with and without surgical intervention, in a large-scale Asian population. Patients hospitalized within 2000 to 2013 who were newly diagnosed with sICH were identified using the National Health Insurance Research Database of Taiwan. Neuroendoscopy and craniotomy groups comprised patients who underwent surgical treatment within 1 week, while those in the control group did not undergo early surgical treatment. Outcomes included subsequent hemorrhagic and ischemic stroke, following acute myocardial infarction, congestive heart failure, and mortality. After propensity score matching, there were 663 patients in each group. Compared to that in the control group, the neuroendoscopy and craniotomy groups had a significantly higher risk of secondary vascular events at 1 to 3 months of follow-up (adjusted HR, 2.08 and 1.95; 95\% CI, 1.21-3.58 and 1.13-3.35; $p<0.01$ and $p<0.05$, respectively), but a significantly lower risk after 3 years of follow-up (adjusted HR, 0.52 and 0.52 ; 95\% CI, 0.35 0.78 and $0.35-0.77 ; p<0.01$ and $p<0.01$, respectively). The mortality rate was higher in the craniotomy group at 6 to 12 months of follow-up (adjusted HR, 2.18; 95\% CI, 1.06-4.49; $p<0.05$ ) compared to that in the control group. Thus, a timely surgical intervention for hematoma evacuation is advantageous in preventing secondary vascular events and improving outcomes in the long term. However, greater attention to secondary ischemic stroke following the initial sICH episode is needed.
\end{abstract}

Key Words Spontaneous intracerebral hemorrhage $\cdot$ neuroendoscopy $\cdot$ craniotomy $\cdot$ subsequent vascular risk $\cdot$ stroke $\cdot$ mortality

XianXiu Chen and Chih-ying Wu are joint first authors. Chih-ying Wu contributed equally to the work.

Cheng-Di Chiu

cdchiu4046@gmail.com

1 Chinese Medicine Research Center, China Medical University, Taichung 404, Taiwan

2 Research Center for Chinese Herbal Medicine, China Medical University, Taichung 404, Taiwan

3 School of Medicine, China Medical University, Taichung, Taiwan

4 Management Office for Health Data, China Medical University Hospital, Taichung, Taiwan

5 Department of Neurosurgery, China Medical University Hospital, 2 Hsueh-Shuh Road, Taichung 40407, Taiwan
6 Stroke Center, China Medical University Hospital, Taichung, Taiwan

7 The Graduate Institute of Integrated Medicine, China Medical University, Taichung, Taiwan

8 Department of Internal Medicine, China Medical University Hospital, Taichung, Taiwan

9 Department of Health Services Administration, China Medical University, Taichung, Taiwan

10 Graduate Institute of Biomedical Science, China Medical University, Taichung, Taiwan 


\section{Introduction}

\section{Background}

Spontaneous intracerebral hemorrhage ( $\mathrm{sICH})$ is devastating and deadly, with 30-day and 1-year mortality rates of approximately $40 \%$ [1] and 54\% [2], respectively. Furthermore, longterm survivors are often saddled with permanent deficits, with up to $75 \%$ of survivors suffering significant disability [3]. Thus, sICH is a sudden event with an extremely high rate of mortality in the short term and disability in the long term. In addition, the burden of hemorrhagic stroke has increased by 47\% between 1990 and 2010 [4]. As the outcomes are poor, timely medical treatment is critical, and optimal management is a priority. However, no known perfect and absolutely effective interventions are currently available for sICH [5]. Although most patients can be managed conservatively, surgical evacuation of the hematoma is immediately required for patients with extensive intracerebral hemorrhage (ICH) [6, 7]. However, the most beneficial surgical procedures for $\mathrm{sICH}$ patients remain controversial.

\section{Objectives}

We aimed to evaluate the risk of subsequent vascular disease and mortality in patients newly diagnosed with acute sICH who underwent surgical treatment (neuroendoscopy or craniotomy evacuation) compared to that in patients who did not undergo surgical treatment.

\section{Methods}

\section{Study Design, Setting, and Patients}

A nationwide, cohort, retrospective, population-based, Taiwanese-registry study was conducted using the National Health Insurance Research Database (NHIRD) of Taiwan. Clinical diagnoses are coded in the NHIRD using the International Classification of Diseases, Ninth Revision, Clinical Modification (ICD-9-CM). The study population comprised inpatients with a claim for a hospitalization between 1 January 2000 and 31 December 2013, indicating a diagnosis of ICH (ICD-9-CM: 431), which was defined as the index event. Patients who underwent computed tomography (CT) or magnetic resonance imaging (MRI) within 2 days (before or after) the hospitalization date, which was defined as the index date, were included. Patients with any diagnosis of ICH before the index date were excluded, unless they were diagnosed within $48 \mathrm{~h}$ before the index date. Patients with any trauma (ICD-9-CM: 800-929) in the 2 weeks before the index date, or with a diagnosis of traumatic brain injury (TBI; ICD9-CM: 85X) after the index date, were excluded.
Patients who underwent neuroendoscopy evacuation within 1 week of the diagnosis of sICH were categorized into the neuroendoscopy group. Patients who underwent craniotomy evacuation within 1 week of the diagnosis of sICH were categorized into the craniotomy group. Patients who did not undergo neuroendoscopy or craniotomy within 1 week of the diagnosis of sICH were defined as the control group. Patients who underwent both neuroendoscopy and craniotomy within 1 week of the diagnosis of sICH were not enrolled, as such cases were limited in number (i.e., their statistical significance may be omitted) and complicated factors, such as whether neuroendoscopy or craniotomy was performed first, would render simple comparisons between surgery (endoscopic or craniotomy) and medical treatment difficult.

\section{Data Source}

The NHIRD is derived from the claims data of the National Health Insurance program, which is a mandatory-enrollment, single-payment system created in 1995 that covers over $99 \%$ of Taiwan's population $[8,9]$. The NHIRD provides an opportunity to evaluate treatment outcomes and drug-disease associations in longitudinal studies $[10,11]$. Specifically compiled for research purposes, this database originates from administrative databases, whose main function is for insurance claim reimbursement; thus, unknown or questionable validity regarding clinical information could exist. However, databases such as the NHIRD must be consistent with the original clinical data source on key elements; an attribute (e.g., drug exposure or diagnosis) in an administrative database for a particular patient should be noted (true positive) in the original clinical records and vice versa (true negative) $[12,13]$. The data quality regarding the diagnosis of stroke has been systematically evaluated and was found to be highly accurate, with acceptable validity for the research of stroke [14]. To protect personal information, patient data were de-identified before the inpatient database was released [8]; thus, informed patient consent was waived for the present study. This study was approved by the Institutional Review Board of China Medical University and Hospital in Taiwan (approval no. CMUH-104-REC2-115).

\section{Outcomes}

The outcome variables are the subsequent vascular events which comprised cerebrovascular events, including hemorrhagic stroke (HS; ICD-9-CM: 430-432) and ischemic stroke (IS; ICD-9-CM: 433-436); cardiovascular events, including acute myocardial infarction (AMI; ICD-9-CM: 410); congestive heart failure (CHF; ICD-9-CM: 428); and mortality. Baseline comorbidities (medical history), including previous IS (ICD-9-CM: 433-436), coronary artery disease (CAD; ICD-9-CM: 411-414), hypertension (HT; ICD-9-CM: 401- 
405), atrial fibrillation (AF; ICD-9-CM: 427.31), hyperlipidemia (HL; ICD-9-CM: 272.0-272.4), diabetes mellitus (DM; ICD-9-CM: 250), chronic obstructive pulmonary disease (COPD; ICD-9-CM: 491, 492, 496), and chronic kidney disease (CKD; ICD-9-CM: 250.4, 274.1, 283.11, 403, 404, $440.1,442.1,447.3,572.4,580-588,642.1,646.2)$, were noted.

\section{Statistical Methods}

Propensity score matching (1:1) on age, sex, index year, and comorbidities was performed. After matching, group differences were evaluated using the chi-square test for categorical variables and the analysis of variance (ANOVA) for age. Adjusted hazard ratios (aHRs) were calculated using Cox proportional hazards regression models, adjusting for sex, age, comorbidities, and the occurrence of outcome events within 1 month after the index date, stratified by the follow-up period. The cumulative incidence of the outcomes was estimated using the Kaplan-Meier method, and the log-rank test was used to evaluate group differences. All tests were 2-sided, and $p$ values $<0.05$ were considered statistically significant. All analyses were performed using SAS (version 9.4; SAS Institute, Inc., Cary, NC).

\section{Results}

\section{Participants}

We identified 60,703 patients with a new diagnosis of sICH and imaging evidence within 2 days (before or after) of the index event. After excluding patients with a record of trauma 2 weeks before the index date or a record of TBI after the index date, as well as patients who underwent both neuroendoscopy and craniotomy, a total of 59,399 patients remained. After 1:1 matching on age, sex, index year, and comorbidities, there were 663 patients in each group (control, neuroendoscopy, and craniotomy).

\section{Descriptive Data}

Baseline characteristics and the mean follow-up periods are provided in Table 1 . There were no statistically significant differences among the groups. However, there was a significant difference in endotracheal tube insertion during hospitalization for the initial sICH among the groups $(p<0.001)$.

\section{Risk of All Secondary Vascular Events and Mortality (Fig. 1A, G)}

Table 2 presents the aHRs and 95\% confidence intervals (CIs) from the Cox proportional hazards regression models. At 1 to
3 months of follow-up, the risk of all secondary vascular events (subsequent HS, IS, AMI, CHF) was significantly higher in the neuroendoscopy (aHR, 2.08; 95\% CI, 1.213.58 ; $p<0.01$ ) and craniotomy (aHR, 1.95; 95\% CI, 1.13$3.35 ; p<0.05)$ groups than in the control group. However, after 3 years of follow-up, the risk of all secondary vascular events was significantly lower in the neuroendoscopy (aHR, $0.52 ; 95 \% \mathrm{CI}, 0.35-0.78 ; p<0.01$ ) and craniotomy (aHR, $0.52 ; 95 \% \mathrm{CI}, 0.35-0.77 ; p<0.01)$ groups than in the control group. In addition, the mortality rate was higher in the craniotomy group at 6 to 12 months of follow-up (aHR, 2.18; 95\% CI, $1.06-4.49 ; p<0.05$ ) than in the control group.

\section{Risk of Subsequent Stroke (Fig. 1B-D)}

Table 3 presents the incidence and risk of subsequent stroke following the first sICH episode, according to the intervention groups. At 1 to 3 months of follow-up, the risk of secondary stroke was significantly higher in the neuroendoscopy (aHR, 2.03; 95\% CI, 1.18-3.5; $p<0.05$ ) and craniotomy (aHR, 1.95; $95 \% \mathrm{CI}, 1.14-3.36 ; p<0.05)$ groups than in the control group. However, after 3 years of follow-up, the risk of subsequent stroke was significantly lower in the neuroendoscopy (aHR, $0.5 ; 95 \% \mathrm{CI}, 0.33-0.77 ; p<0.01)$ and craniotomy (aHR, 0.37 ; $95 \%$ CI, $0.23-0.59 ; p<0.001)$ groups than in the control group. Furthermore, the overall risk of subsequent ischemic stroke was lower in the craniotomy group (aHR, 0.59; 95\% CI, $0.4-0.87 ; p<0.01)$ than in the control group, with subsequent ischemic stroke events occurring in 77/663 patients (recurrence rate, 2.49 per 100 person years) in the control group and in 38/663 patients (recurrence rate, 1.48 per 100 person years) in the craniotomy group. Three years after the first sICH episode, the risk of subsequent ischemic stroke decreased further and was significantly lower in the neuroendoscopy (aHR, $0.41 ; 95 \% \mathrm{CI}, 0.22-0.76 ; p<0.01$ ) and craniotomy (adjusted HR, 0.24; 95\% CI, 0.11-0.51; $p<0.001)$ groups than in the control group. In addition, the overall risk of subsequent hemorrhagic stroke was higher in the neuroendoscopy group (aHR, 1.44; 95\% CI, 1.05-1.99; $p<0.05)$ than in the control group, with subsequent hemorrhagic stroke events occurring in 69/663 patients (recurrence rate, 2.23 per 100 person years) in the control group, and in $84 / 663$ patients (recurrence rate, 3.19 per 100 person years) in the neuroendoscopy group. Furthermore, at 1 to 3 months and at 3 to 6 months of follow-up, the risk of recurrent hemorrhagic stroke was higher in the neuroendoscopy group (13 months: aHR, 2.56 ; 95\% CI, $1.32-4.98 ; p<0.01)(3-$ 6 months: adjusted HR, 4.59 95\% CI, $1.29-16.4 ; p<0.05$ ) and craniotomy group (aHR, 2.55; 95\% CI, 1.32-4.93; $p<0.01)$ than in the control group. Additionally, the risk of subsequent hemorrhagic stroke remained higher in the neuroendoscopy group than in the control group at 1 to 2 years of follow-up (aHR, 6.03; 95\% CI, 1.29-28.3; $p<0.05$ ). 
Table 1 Demographics of patients with ICH

\begin{tabular}{|c|c|c|c|c|c|c|c|}
\hline \multirow[t]{3}{*}{ Characteristic } & \multicolumn{6}{|l|}{$\mathrm{ICH}$} & \multirow[t]{3}{*}{$p$ value } \\
\hline & \multicolumn{2}{|c|}{$\begin{array}{l}\text { Control } \\
(N=663)\end{array}$} & \multicolumn{2}{|c|}{$\begin{array}{l}\text { Neuroendoscopy } \\
(N=663)\end{array}$} & \multicolumn{2}{|c|}{$\begin{array}{l}\text { Craniotomy } \\
(N=663)\end{array}$} & \\
\hline & $n$ & $\%$ & $n$ & $\%$ & $n$ & $\%$ & \\
\hline Sex & & & & & & & 0.95 \\
\hline Female & 228 & 34.39 & 232 & 34.99 & 233 & 35.14 & \\
\hline Male & 435 & 65.61 & 431 & 65.01 & 430 & 64.86 & \\
\hline Age, years ${ }^{\dagger}$ & & & & & & & 0.99 \\
\hline$<35$ & 35 & 5.28 & 34 & 5.13 & 34 & 5.13 & \\
\hline $35-64$ & 364 & 54.9 & 367 & 55.35 & 363 & 54.75 & \\
\hline$\geq 65$ & 264 & 39.82 & 262 & 39.52 & 266 & 40.12 & \\
\hline Mean (SD) ${ }^{\#}$ & \multicolumn{2}{|c|}{$60.5(16.1)$} & \multicolumn{2}{|c|}{$59.2(15.1)$} & \multicolumn{2}{|c|}{$59.9(15.4)$} & 0.31 \\
\hline \multicolumn{8}{|l|}{ Baseline comorbidity } \\
\hline Previous ischemic stroke & 73 & 11.01 & 70 & 10.56 & 71 & 10.71 & 0.96 \\
\hline CAD & 32 & 4.83 & 31 & 4.68 & 39 & 5.88 & 0.55 \\
\hline Hypertension & 182 & 27.45 & 179 & 27 & 178 & 26.85 & 0.97 \\
\hline Atrial fibrillation & 22 & 3.32 & 21 & 3.17 & 18 & 2.71 & 0.80 \\
\hline Hyperlipidemia & 34 & 5.13 & 33 & 4.98 & 39 & 5.88 & 0.73 \\
\hline Diabetes mellitus & 76 & 11.46 & 72 & 10.86 & 84 & 12.67 & 0.58 \\
\hline COPD & 30 & 4.52 & 33 & 4.98 & 32 & 4.83 & 0.93 \\
\hline CKD & 20 & 3.02 & 23 & 3.47 & 22 & 3.32 & 0.89 \\
\hline Endotracheal tube insertion & 93 & 14.03 & 147 & 22.17 & 130 & 19.61 & $<0.001$ \\
\hline Mean admission days (median) & \multicolumn{2}{|c|}{$17(11)$} & \multicolumn{2}{|c|}{$23(24)$} & \multicolumn{2}{|c|}{$23(21)$} & \\
\hline Mean follow-up period (median) & \multicolumn{2}{|c|}{$4.67(3.64)$} & \multicolumn{2}{|c|}{$3.97(1.91)$} & \multicolumn{2}{|c|}{$3.86(1.91)$} & \\
\hline
\end{tabular}

$\mathrm{SD}=$ standard deviation $\mathrm{CAD}=$ coronary artery disease $\mathrm{COPD}=$ chronic obstructive pulmonary disease $\mathrm{CKD}=$ chronic kidney disease

${ }^{\dagger}$ Chi-square tests

${ }^{\text {\# }}$ ANOVA

However, the risk of subsequent hemorrhagic stroke decreased with time and was significantly lower in the craniotomy group (aHR, $0.51 ; 95 \% \mathrm{CI}, 0.28-0.95 ; p<0.05)$ than in the control group after 3 years of follow-up.

\section{Subsequent Acute Myocardial Infarction and Congestive Heart Failure (Fig. 1E, F)}

Table 4 presents the incidence and risk of AMI and CHF following the initial sICH episode, according to the intervention groups. There were no statistically significant differences among these 3 groups.

\section{Discussion}

\section{Key Results}

The present study evaluated subsequent vascular risk (a composite of HS, IS, AMI, and CHF) and mortality in patients with a new diagnosis of acute sICH who underwent neuroendoscopy evacuation, craniotomy evacuation, or neither. Four major findings were noted. First, mortality increased in patients who underwent craniotomy versus those who did not in the perioperative period. Second, patients who underwent neuroendoscopy or craniotomy had a higher risk of secondary vascular events (IS, HS, AMI, CHF) during the perioperative period compared to that in patients who did not undergo surgical treatment, with the highest risk in those who underwent neuroendoscopy. However, the subsequent vascular risk decreased over time in both the neuroendoscopy and craniotomy groups, becoming lower than that in the control group after 3 years of follow-up, with the lowest risk of subsequent stroke (IS, HS) in those who underwent craniotomy. Third, both the neuroendoscopy and craniotomy groups had a lower risk of developing secondary IS compared to that in the control group, with the lowest risk in the craniotomy group. Fourth, both the neuroendoscopy and craniotomy groups had a higher risk of developing secondary HS during the perioperative period compared to that in the control group, 
Table 2 All outcomes and mortality, stratified by follow-up period

\begin{tabular}{|c|c|c|c|c|c|c|c|c|c|c|c|c|}
\hline \multirow{3}{*}{$\begin{array}{l}\text { Follow-up } \\
\text { period }\end{array}$} & \multicolumn{9}{|l|}{$\mathrm{ICH}$} & \multirow{3}{*}{$\begin{array}{l}\text { Neuroendoscopy } \\
\text { versus control } \\
\mathrm{aHR}^{\dagger}(95 \% \mathrm{CI})\end{array}$} & \multirow{3}{*}{$\begin{array}{l}\text { Craniotomy } \\
\text { versus control } \\
\mathrm{aHR}^{\dagger}(95 \% \mathrm{CI})\end{array}$} & \multirow{3}{*}{$\begin{array}{l}\text { Neuroendoscopy } \\
\text { versus craniotomy } \\
\text { aHR }^{\dagger}(95 \% \text { CI) }\end{array}$} \\
\hline & \multicolumn{3}{|c|}{ Control } & \multicolumn{3}{|c|}{ Neuroendoscopy } & \multicolumn{3}{|c|}{ Craniotomy } & & & \\
\hline & $\begin{array}{l}\text { Event } \\
\text { no. }\end{array}$ & PY & Rate & $\begin{array}{l}\text { Event } \\
\text { no. }\end{array}$ & PY & Rate & $\begin{array}{l}\text { Event } \\
\text { no. }\end{array}$ & PY & Rate & & & \\
\hline \multicolumn{13}{|l|}{ All outcomes } \\
\hline Overall & 162 & 3097 & 5.23 & 138 & 2635 & 5.24 & 129 & 2560 & 5.04 & $1.02(0.81-1.28)$ & $0.94(0.74-1.18)$ & $1.09(0.85-1.38)$ \\
\hline $1-3$ months & 21 & 136 & 15.5 & 37 & 128 & 28.8 & 36 & 130 & 27.7 & $2.08(1.21-3.58)^{* *}$ & $1.95(1.13-3.35)^{*}$ & $1.07(0.67-1.7)$ \\
\hline 3-6 months & 13 & 121 & 10.8 & 16 & 108 & 14.8 & 13 & 110 & 11.8 & $1.43(0.68-3)$ & $1.1(0.51-2.39)$ & $1.3(0.62-2.71)$ \\
\hline 6-12 months & 12 & 228 & 5.26 & 15 & 196 & 7.65 & 15 & 199 & 7.55 & $1.42(0.66-3.06)$ & $1.42(0.66-3.05)$ & $1(0.49-2.05)$ \\
\hline $1-2$ years & 15 & 416 & 3 & 16 & 351 & 4.56 & 16 & 351 & 4.56 & $1.39(0.68-2.82)$ & $1.27(0.62-2.58)$ & $1.1(0.55-2.2)$ \\
\hline $2-3$ years & 20 & 370 & 5.41 & 18 & 309 & 5.82 & 13 & 309 & 4.21 & $1.12(0.59-2.12)$ & $0.74(0.37-1.5)$ & $1.5(0.73-3.08)$ \\
\hline$>3$ years & 81 & 1828 & 4.43 & 36 & 1543 & 2.33 & 36 & 1462 & 2.46 & $0.52(0.35-0.78)^{* *}$ & $0.52(0.35-0.77)^{* *}$ & $1.01(0.63-1.61)$ \\
\hline \multicolumn{13}{|l|}{ Mortality } \\
\hline Overall & 203 & 3792 & 5.35 & 186 & 3345 & 5.56 & 225 & 3198 & 7.03 & $1.1(0.89-1.34)$ & $1.2(0.99-1.45)$ & $0.91(0.75-1.12)$ \\
\hline $1-3$ months & 64 & 138 & 46.5 & 66 & 132 & 49.8 & 88 & 133 & 66 & $1.03(0.73-1.46)$ & $1.36(0.98-1.87)$ & $0.76(0.55-1.05)$ \\
\hline 3-6 months & 10 & 128 & 7.84 & 7 & 119 & 5.87 & 13 & 121 & 10.8 & $0.87(0.33-2.3)$ & $1.49(0.65-3.43)$ & $0.58(0.23-1.47)$ \\
\hline 6-12 months & 11 & 244 & 4.51 & 15 & 225 & 6.67 & 23 & 223 & 10 & $1.58(0.73-3.46)$ & $2.18(1.06-4.49)^{*}$ & $0.73(0.38-1.4)$ \\
\hline $1-2$ years & 21 & 457 & 4.6 & 24 & 415 & 5.78 & 20 & 409 & 4.89 & $1.37(0.76-2.47)$ & $0.98(0.53-1.82)$ & $1.4(0.77-2.55)$ \\
\hline $2-3$ years & 18 & 421 & 4.27 & 10 & 377 & 2.65 & 18 & 373 & 4.82 & $0.69(0.32-1.5)$ & $0.96(0.49-1.88)$ & $0.72(0.32-1.59)$ \\
\hline$>3$ years & 79 & 2404 & 3.29 & 64 & 2076 & 3.08 & 63 & 1939 & 3.25 & $1.06(0.76-1.48)$ & $0.99(0.71-1.39)$ & $1.07(0.75-1.53)$ \\
\hline
\end{tabular}

Rate indicates the incidence rate or mortality rate (per 100 person years)

aHR = adjusted hazard ratio; $\mathrm{CI}=$ confidence interval; $\mathrm{PY}=$ person years

${ }^{\dagger}$ Adjusted HR: adjusted for sex, age, comorbidities, previous ischemic stroke, and outcome events within 1 month post-index date in Cox proportional hazards regression models

${ }^{*} p<0.05 ;{ }^{* *} p<0.01$

and the risk remained higher in the neuroendoscopy group than in the control group for 2 years of follow-up. However, the risk of secondary HS decreased over time, becoming significantly lower in the craniotomy group compared to that in the control group after 3 years of follow-up.

\section{Limitations}

The main strengths of the present study include the large nationwide sample and comprehensive demographic characteristics. However, this was a retrospective secondary data analysis; several limitations exist and should be acknowledged. First, data on factors that may be related to the severity of the stroke and would directly affect the sICH prognosis, such as the initial hematoma volume and location, initial severity score (e.g., NIH Stroke Scale (NIHSS)), Glasgow Coma Scale (GCS), modified Rankin Scale (mRS), and Breidbart Index (BI), were lacking. Unfortunately, the NHIRD does not have this information. Thus, the cohorts may have differed in terms of the preoperative neurological status, degree of neurological impairment, level of consciousness, experience of the surgeon, and so on, which raises concerns regarding substantial unaccounted confounding. However, we enrolled only patients hospitalized for sICH who underwent imaging within 2 days of the index date, which would focus the severity somewhat. Furthermore, we evaluated the endotracheal tube insertion status and hospitalization days of the initial sICH, which might be related to the initial severity. Second, there was a lack of information regarding the medication history; however, data regarding the use of antithrombotic agents was unavailable. Third, it is unclear whether rebleeding/ secondary ICH and mortality after surgery were due to the risks of the initial ICH itself or a surgical complication, as the present study cannot address the cause. In addition, we could not clearly determine the rationale for the choice of craniotomy or endoscopic approach as the surgical procedures. Although most neurosurgeons in Taiwan would choose craniotomy rather than endoscopic evacuation in patients suffering from greater mass effect, more midline shift and a more diffused hemorrhage are similar to other documentations [15]. Lastly, even though a population-based database was utilized, we did not use randomization and lack adequate control variables, which may have introduced a 

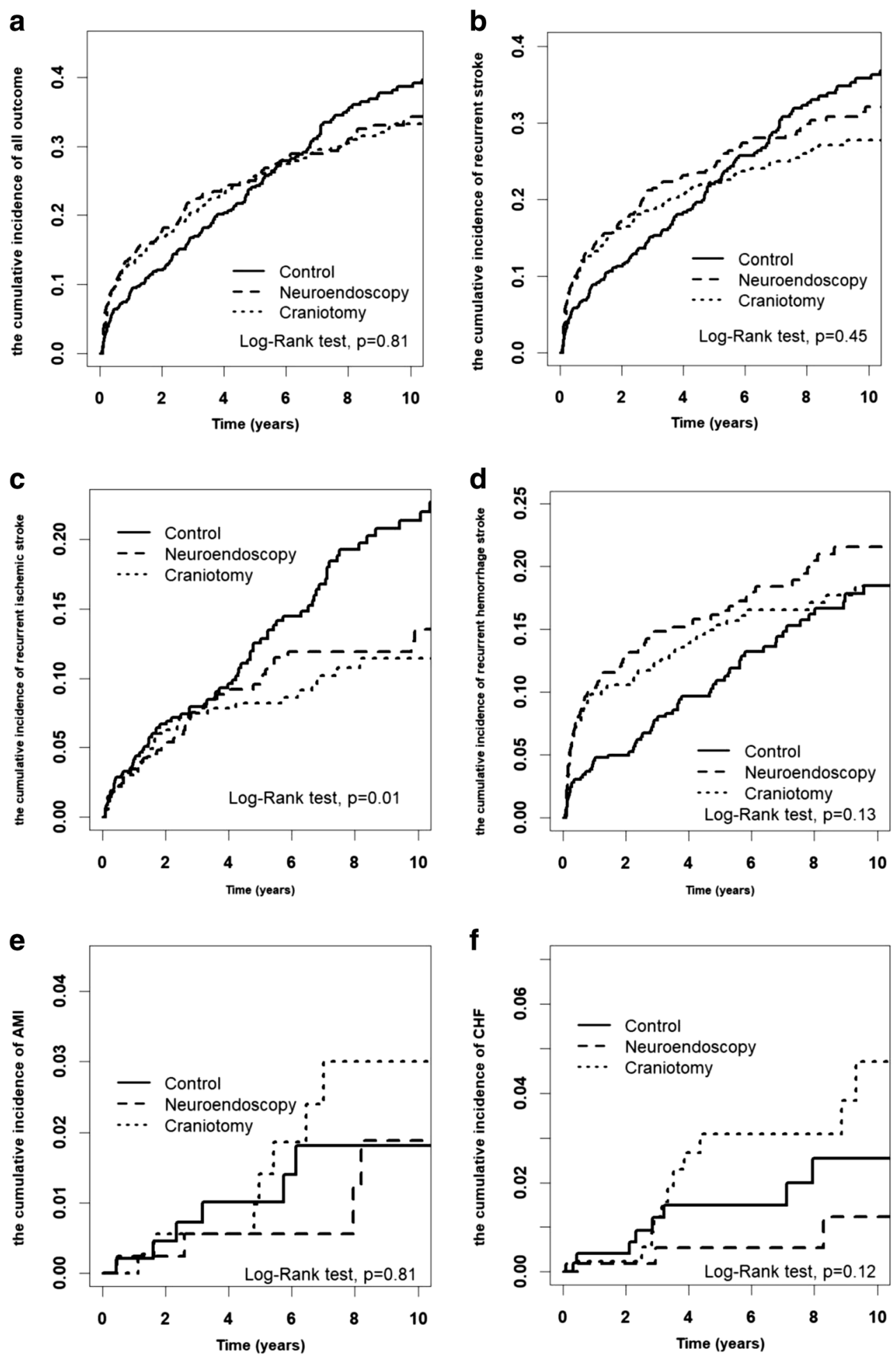

f

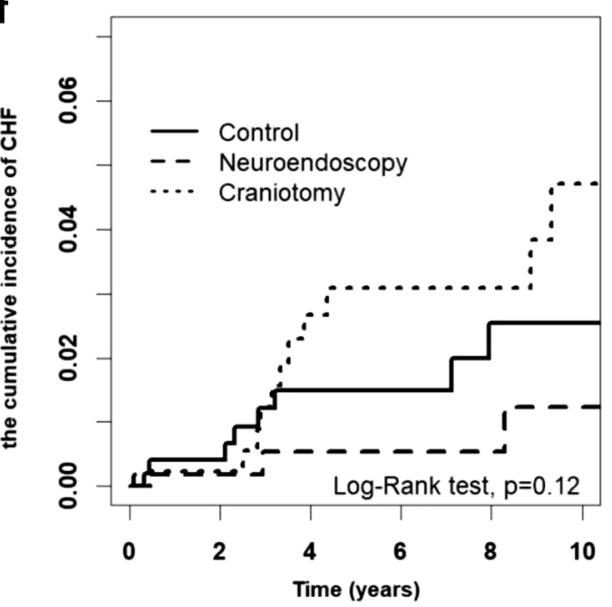

Fig. 1 Cumulative hazard ratio and cumulative incidences after the initial onset in patients with a new diagnosis of spontaneous intracerebral hemorrhage (sICH). A Cumulative incidence of all secondary vascular outcomes (hemorrhagic stroke, ischemic stroke, acute myocardial infarction (AMI), and congestive heart failure (CHF)) after the initial ICH onset. B Cumulative incidence of all secondary stroke outcomes (hemorrhagic stroke and ischemic stroke) after the initial ICH onset. C

Cumulative incidence of subsequent ischemic stroke after the initial ICH onset. D Cumulative incidence of subsequent hemorrhagic stroke after the initial ICH onset. E Cumulative incidence of subsequent acute myocardial infarction after the initial ICH onset. F Cumulative incidence of subsequent congestive heart failure after the initial ICH onset. G Cumulative incidence of subsequent mortality after the initial $\mathrm{ICH}$ onset. 


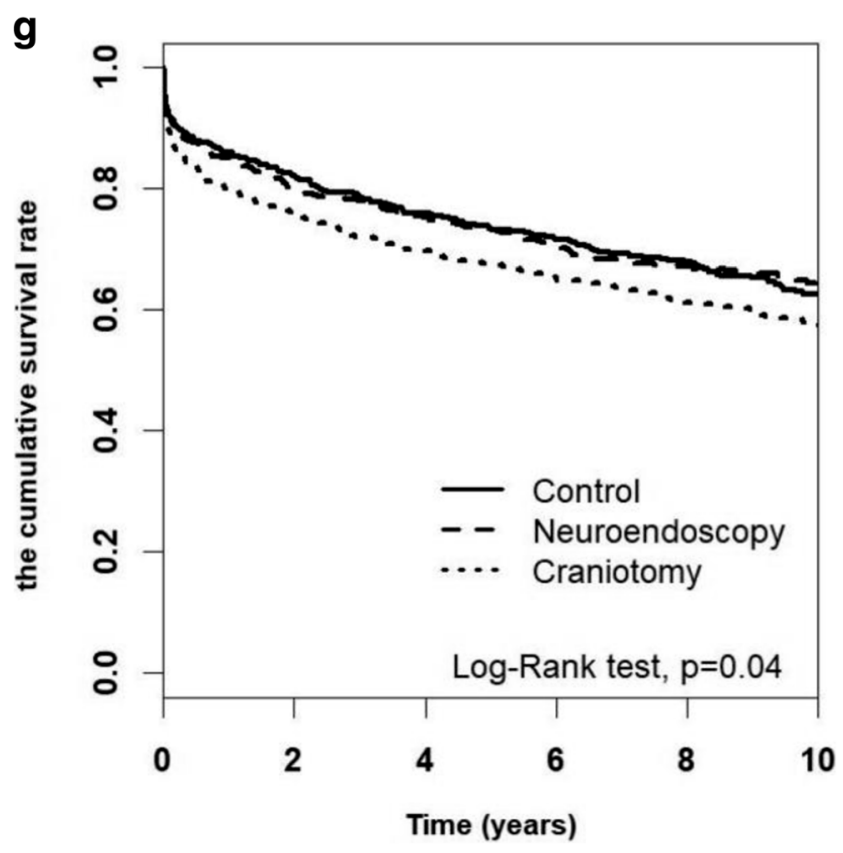

Fig. 1 (continued)

selection bias, rendering it harder to interpret the observed associations. Furthermore, awareness of the limitations inherent in national databases that are intended for a purpose other than clinical studies (e.g., insurance) is required.

\section{Interpretation}

One of the most famous clinical trials for the surgical treatment of sICH (the STICH trial) suggested no benefit for early hematoma evacuation compared to conservative medical treatment except in some subgroups of patients $[16,17]$. As advances in surgical skills and tools have accumulated, more and more minimally invasive procedures have taken the place of craniotomy in the treatment of sICH. There are several ongoing randomized trails, including the Minimally Invasive Surgery Plus Alteplase in ICH Evacuation (MISTIE) trial. The MISTIE phase II trial showed a trend towards improved outcomes in the surgical patients compared to those in the medically managed patients. In addition, the ongoing MISTIE III trial added a stereotactic CT-guided endoscopic surgery arm [18-21]. In the present study, we attempted to compare the short-term and long-term effectiveness of sICH treatment, including medical treatment (control) and surgical intervention (craniotomy and endoscope). Our results suggested an advantage of timely surgical intervention for hematoma evacuation, as it may prevent secondary vascular events in the long term.
Many previous animal and clinical studies indicated that brain edema, blood-brain barrier disruption, and increased intracranial pressure (ICP) result not only from mass effect; blood component exposure, such as thrombin, heme, and so on, further deteriorates the secondary injury $[22,23]$. In addition, elevated ICP is the leading cause of mortality in patients with sICH in the hyperacute (first $48 \mathrm{~h}$ ) time window [24]. Early surgical intervention in critical patients, regardless of the manner of the craniotomy or endoscopic approach, can reduce mass effect, eliminate blood component exposure, and lower ICP. However, there are still many debates on when and how surgical intervention should be done [25]. In the present study, we only classified surgical groups into a minimally invasive endoscopic approach group and an open surgery group, which included craniotomy or craniectomy approaches for hematoma evacuation. Traditionally, it is taken for granted that open surgery, compared with the endoscopic approach, can offer better mass effect reduction, clearer view, and more complete hematoma evacuation and irrigation around damaged tissues [15]. Our findings suggest that the endoscopic group is not superior in shortterm or long-term mortality, secondary vascular events, subsequent stroke, acute myocardial infarction, and congestive heart failure compared to the craniotomy group (Tables 2, 3, and 4).

In the present study, we noted that the surgical groups possessed a higher risk of secondary vascular events at 1 to 3 months of follow-up, which may be directly or indirectly related to the surgical procedures [26]. On the other hand, most patients who received surgical intervention might be more severe in their initial conditions. The short-term unstable blood-brain barrier caused by either surgery or a larger volume of hematoma destruction may result in the increased risk of vascular events within the first 3 months. The present findings echoed a trade-off between a high risk of rebleeding during the perioperative period and significantly decreased subsequent stroke in the long term.

As a "stroke-prone" state may present in either hemorrhagic or ischemic stroke, secondary vascular events may be provoked by several factors after the initial sICH [27]. But, why would surgical intervention groups have a lower incidence of vascular events for as long as 3 years? As most of the patients with sICH may have received surgical intervention because of a clinically larger hematoma and more severe mass effect, we suspect that the patients in the surgery group who survived over 3 years did so not only because of a potentially better physical condition before events but also because of closer follow-ups and/or more 
Table 3 Incidence and risk of recurrent stroke, stratified by follow-up period

\begin{tabular}{|c|c|c|c|c|c|c|c|c|c|}
\hline \multirow{3}{*}{$\begin{array}{l}\text { Follow-up } \\
\text { period }\end{array}$} & \multicolumn{6}{|l|}{$\mathrm{ICH}$} & \multirow{3}{*}{$\begin{array}{l}\text { Neuroendoscopy } \\
\text { versus control } \\
\operatorname{aHR}^{\dagger}(95 \% \mathrm{CI})\end{array}$} & \multirow{3}{*}{$\begin{array}{l}\text { Craniotomy } \\
\text { versus control } \\
\text { aHR }(95 \% \mathrm{CI})\end{array}$} & \multirow{3}{*}{$\begin{array}{l}\text { Neuroendoscopy } \\
\text { versus craniotomy } \\
\mathrm{aHR}^{\dagger}(95 \% \mathrm{CI})\end{array}$} \\
\hline & Control & & Neuroendosc & & Craniotomy & & & & \\
\hline & $\begin{array}{l}\text { Event PY } \\
\text { no. }\end{array}$ & $\mathrm{RR}$ & $\begin{array}{l}\text { Event PY } \\
\text { no. }\end{array}$ & $\mathrm{RR}$ & $\begin{array}{l}\text { Event PY } \\
\text { no. }\end{array}$ & $\mathrm{RR}$ & & & \\
\hline
\end{tabular}

\begin{tabular}{|c|c|c|c|c|c|c|c|c|c|c|c|c|}
\hline \multicolumn{13}{|c|}{ Stroke (ICD-9-CM: 431-436) } \\
\hline Overall & 146 & 3097 & 4.71 & 129 & 2635 & 4.89 & 110 & 2560 & 4.3 & $1.06(0.83-1.34)$ & $0.89(0.7-1.15)$ & $1.18(0.92-1.53)$ \\
\hline $1-3$ months & 21 & 136 & 15.5 & 36 & 128 & 28.1 & 36 & 130 & 27.7 & $2.03(1.18-3.5)^{*}$ & $1.95(1.14-3.36)^{*}$ & $1.04(0.65-1.65)$ \\
\hline $3-6$ months & 10 & 121 & 8.29 & 15 & 108 & 13.9 & 12 & 110 & 10.9 & $1.73(0.77-3.89)$ & $1.33(0.57-3.09)$ & $1.3(0.61-2.79)$ \\
\hline 6-12 months & 12 & 228 & 5.26 & 15 & 196 & 7.65 & 15 & 199 & 7.55 & $1.42(0.66-3.06)$ & $1.42(0.66-3.05)$ & $1(0.49-2.05)$ \\
\hline $1-2$ years & 14 & 416 & 3.37 & 16 & 351 & 4.56 & 14 & 351 & 3.99 & $1.46(0.71-3)$ & $1.18(0.56-2.48)$ & $1.24(0.6-2.55)$ \\
\hline $2-3$ years & 16 & 370 & 4.33 & 16 & 309 & 5.18 & 10 & 309 & 3.24 & $1.26(0.63-2.54)$ & $0.72(0.32-1.6)$ & $1.75(0.79-3.89)$ \\
\hline$>3$ years & 73 & 1828 & 3.99 & 31 & 1543 & 2.01 & 23 & 1462 & 1.57 & $0.5(0.33-0.77) * *$ & $0.37(0.23-0.59)^{* * *}$ & $1.35(0.79-2.32)$ \\
\hline \multicolumn{13}{|c|}{ Ischemic stroke (ICD-9-CM: 433-436) } \\
\hline Overall & 77 & 3097 & 2.49 & 45 & 2635 & 1.71 & 38 & 2560 & 1.48 & $0.71(0.49-1.03)$ & $0.59(0.4-0.87)^{* *}$ & $1.21(0.79-1.87)$ \\
\hline $1-3$ months & 8 & 136 & 5.9 & 8 & 128 & 6.23 & 7 & 130 & 5.39 & $1.2(0.44-3.24)$ & $1.02(0.37-2.83)$ & $1.18(0.42-3.27)$ \\
\hline 3-6 months & 7 & 121 & 5.8 & 3 & 108 & 2.77 & 3 & 110 & 2.72 & $0.52(0.13-2.07)$ & $0.43(0.11-1.73)$ & $1.21(0.24-6.11)$ \\
\hline 6-12 months & 5 & 228 & 2.19 & 5 & 196 & 2.55 & 5 & 199 & 2.52 & $1.16(0.33-4.08)$ & $1.09(0.31-3.84)$ & $1.07(0.31-3.71)$ \\
\hline $1-2$ years & 12 & 416 & 2.89 & 7 & 351 & 2 & 11 & 351 & 3.14 & $0.74(0.29-1.89)$ & $1.06(0.47-2.43)$ & $0.7(0.27-1.8)$ \\
\hline $2-3$ years & 5 & 370 & 1.35 & 8 & 309 & 2.59 & 4 & 309 & 1.3 & $1.92(0.62-5.95)$ & $0.81(0.21-3.09)$ & $2.36(0.7-7.99)$ \\
\hline$>3$ years & 40 & 1828 & 2.19 & 14 & 1543 & 0.91 & 8 & 1462 & 0.55 & $0.41(0.22-0.76)^{* *}$ & $0.24(0.11-0.51)^{* * *}$ & $1.71(0.71-4.08)$ \\
\hline \multicolumn{13}{|c|}{ Hemorrhage stroke (ICD-9-CM: 431-432) } \\
\hline Overall & 69 & 3097 & 2.23 & 84 & 2635 & 3.19 & 72 & 2560 & 2.81 & $1.44(1.05-1.99) *$ & $1.24(0.89-1.72)$ & $1.17(0.85-1.6)$ \\
\hline $1-3$ months & 13 & 136 & 9.59 & 28 & 128 & 21.8 & 29 & 130 & 22.3 & $2.56(1.32-4.98)^{* *}$ & $2.55(1.32-4.93)^{* *}$ & $1(0.6-1.69)$ \\
\hline $3-6$ months & 3 & 121 & 2.49 & 12 & 108 & 11.1 & 9 & 110 & 8.17 & $4.59(1.29-16.4)^{*}$ & $3.37(0.91-12.5)$ & $1.36(0.57-3.24)$ \\
\hline 6-12 months & 7 & 228 & 3.07 & 10 & 196 & 5.1 & 10 & 199 & 5.03 & $1.53(0.58-4.04)$ & $1.6(0.61-4.2)$ & $0.96(0.4-2.32)$ \\
\hline $1-2$ years & 2 & 416 & 0.48 & 9 & 351 & 2.57 & 3 & 351 & 0.86 & $6.03(1.29-28.3)^{*}$ & $1.77(0.29-10.7)$ & $3.41(0.91-12.8)$ \\
\hline $2-3$ years & 11 & 370 & 2.98 & 8 & 309 & 2.59 & 6 & 309 & 1.94 & $0.95(0.38-2.4)$ & $0.68(0.25-1.85)$ & $1.41(0.49-4.08)$ \\
\hline$>3$ years & 33 & 1828 & 1.81 & 17 & 1543 & 1.1 & 15 & 1462 & 1.03 & $0.6(0.33-1.08)$ & $0.51(0.28-0.95)^{*}$ & $1.17(0.58-2.36)$ \\
\hline
\end{tabular}

aHR = adjusted hazard ratio; $\mathrm{CI}=$ confidence interval; $\mathrm{PY}=$ person years; $\mathrm{RR}=$ recurrence rate (per 100 person years)

${ }^{\dagger}$ Adjusted HR: adjusted for sex, age, comorbidities, previous ischemic stroke, and outcome events within 1 month post-index date in Cox proportional hazards regression models

$* p<0.05 ; * *<0.01 ; * * *<0.001$

aggressive management of vascular risk factors as a result of their intervention/critically ill status.

\section{Generalizability}

When taking all pros and cons into consideration, this retrospective cohort study suggests a general window of risks and results for the treatment of sICH. Preventing the recurrence of $\mathrm{ICH}$ is the clinical focus after the initial sICH onset; the importance of the control of comorbidities and the management of other following vascular events are well known and, yet, neglected. In the present study, the 3 treatment groups (control, neuroendoscopy, and craniotomy) had the same high ratios of patients with histories of HT, DM, CAD, HL, and COPD, which are all high-risk factors leading to ischemia. Although the present study cannot provide evidence regarding the importance of postoperative blood pressure control and comorbidity management in lowering the risk for subsequent IS in patients with a new diagnosis of $\mathrm{sICH}$, it does call attention to the high risk of secondary IS, especially in those who did not receive surgical intervention.

\section{Conclusions}

This study suggests that timely surgical intervention for hematoma evacuation is advantageous in preventing secondary vascular events and improving outcomes in the long term. Craniotomy remains the most common method for treating $\mathrm{sICH}$, as it affords intraoperative homeostasis and is extraordinarily effective for decompression. With the use of microscopy, craniotomy has additional advantages, such as a good view, allowing the complete clearance of the hematoma, and further improving the outcomes. However, further highquality randomized controlled trials are needed, with patients similar in terms of hematoma volumes, GCS, age, and time of onset to better compare the effects of various surgical interventions on the outcomes in patients with sICH. 
Table 4 Incidence and risk of AMI and CHF, stratified by follow-up period

\begin{tabular}{|c|c|c|c|c|c|c|c|c|c|}
\hline \multirow{3}{*}{$\begin{array}{l}\text { Follow-up } \\
\text { period }\end{array}$} & \multicolumn{6}{|l|}{$\mathrm{ICH}$} & \multirow{3}{*}{$\begin{array}{l}\text { Neuroendoscopy } \\
\text { versus control } \\
\mathrm{aHR}^{\dagger}(95 \% \mathrm{CI})\end{array}$} & \multirow{3}{*}{$\begin{array}{l}\text { Craniotomy } \\
\text { versus control } \\
\text { aHR }(95 \% \mathrm{CI})\end{array}$} & \multirow{3}{*}{$\begin{array}{l}\text { Neuroendoscopy } \\
\text { versus craniotomy } \\
\mathrm{aHR}^{\dagger}(95 \% \mathrm{CI})\end{array}$} \\
\hline & Control & & Neuroendosco & & Craniotomy & & & & \\
\hline & $\begin{array}{l}\text { Event PY } \\
\text { no. }\end{array}$ & IR & $\begin{array}{l}\text { Event PY } \\
\text { no. }\end{array}$ & IR & $\begin{array}{l}\text { Event } \\
\text { no. }\end{array}$ & IR & & & \\
\hline
\end{tabular}

\begin{tabular}{|c|c|c|c|c|c|c|c|c|c|c|c|c|}
\hline \multicolumn{13}{|c|}{ AMI (ICD-9-CM: 410) } \\
\hline Overall & 7 & 3097 & 0.23 & 5 & 2635 & 0.19 & 7 & 2560 & 0.27 & $0.83(0.26-2.65)$ & $1.09(0.38-3.17)$ & $0.76(0.24-2.43)$ \\
\hline $1-3$ months & 0 & 136 & 0 & 0 & 128 & 0 & 0 & 130 & 0 & & & \\
\hline $3-6$ months & 1 & 121 & 0.83 & 1 & 108 & 0.92 & 0 & 110 & 0 & $1.85(0.1-35.8)$ & & \\
\hline 6-12 months & 0 & 228 & 0 & 0 & 196 & 0 & 0 & 199 & 0 & & & \\
\hline $1-2$ years & 1 & 416 & 0.24 & 0 & 351 & 0 & 2 & 351 & 0.57 & & $15.2(0.27-857)$ & \\
\hline $2-3$ years & 1 & 370 & 0.27 & 1 & 309 & 0.32 & 0 & 309 & 0 & & & \\
\hline$>3$ years & 4 & 1828 & 0.22 & 3 & 1543 & 0.19 & 5 & 1462 & 0.34 & $0.85(0.18-4)$ & $1.45(0.37-5.61)$ & $0.58(0.13-2.54)$ \\
\hline \multicolumn{13}{|c|}{ CHF (ICD-9-CM: 428) } \\
\hline Overall & 9 & 3097 & 0.29 & 4 & 2635 & 0.15 & 12 & 2560 & 0.47 & $0.52(0.16-1.72)$ & $1.41(0.58-3.42)$ & $0.37(0.12-1.18)$ \\
\hline $1-3$ months & 0 & 136 & 0 & 1 & 128 & 0.78 & 0 & 130 & 0 & & & \\
\hline $3-6$ months & 2 & 121 & 1.66 & 0 & 108 & 0 & 1 & 110 & 0.91 & & $0.88(0.07-11.43)$ & \\
\hline 6-12 months & 0 & 228 & 0 & 0 & 196 & 0 & 0 & 199 & 0 & & & \\
\hline $1-2$ years & 0 & 416 & 0 & 0 & 351 & 0 & 0 & 351 & 0 & & & \\
\hline $2-3$ years & 3 & 370 & 0.81 & 1 & 309 & 0.32 & 3 & 309 & 0.97 & $0.4(0.04-4.29)$ & $1.29(0.24-6.96)$ & $0.31(0.03-3.09)$ \\
\hline$>3$ years & 4 & 1828 & 0.22 & 2 & 1543 & 0.13 & 8 & 1462 & 0.55 & $0.54(0.09-3.08)$ & $2.07(0.58-7.42)$ & $0.26(0.05-1.3)$ \\
\hline
\end{tabular}

$\mathrm{AMI}=$ acute myocardial infarction; $\mathrm{CHF}=$ congestive heart failure; $\mathrm{aHR}=$ adjusted hazard ratio $\mathrm{CI}=$ confidence interval; $\mathrm{PY}=$ person years; $\mathrm{IR}=$ incidence rate (per 100 person year)

${ }^{\dagger}$ Adjusted HR: adjusted for sex, age, comorbidities, previous ischemic stroke, and outcome events within 1 month post-index date in Cox proportional hazards regression models

\begin{abstract}
Acknowledgments This study was supported by the China Medical University Hospital (DMR-105-054) and was partially supported by the Taiwan Ministry of Health and Welfare Clinical Trial Center (MOHW108-TDU-B-212-133004); China Medical University Hospital; Academia Sinica Stroke Biosignature Project (BM10701010021); MOST Clinical Trial Consortium for Stroke (MOST 107-2321-B-039-004); Tseng-Lien Lin Foundation, Taichung, Taiwan; Katsuzo and Kiyo Aoshima Memorial Funds, Japan; and the "Chinese Medicine Research Center, China Medical University," from the Featured Areas Research Center Program within the framework of the Higher Education Sprout Project by the Ministry of Education (MOE) in Taiwan (CMRC-CMA-7).
\end{abstract}

Role of the Funder/Sponsor The funders had no role in the study design, data collection and analysis, decision to publish, or preparation of the manuscript.

Required Author Forms Disclosure forms provided by the authors are available with the online version of this article.

\section{Compliance with Ethical Standards}

Conflict of Interest The authors declare that they have no conflicts of interest.

Publisher's Note Springer Nature remains neutral with regard to jurisdictional claims in published maps and institutional affiliations.

\section{References}

1. van Asch CJ, Luitse MJ, Rinkel GJ, van der Tweel I, Algra A, Klijn CJ. Incidence, case fatality, and functional outcome of intracerebral haemorrhage over time, according to age, sex, and ethnic origin: a systematic review and meta-analysis. Lancet Neurol 2010;9:167-176.

2. Poon MT, Fonville AF, Al-Shahi Salman R. Long-term prognosis after intracerebral haemorrhage: systematic review and meta-analysis. J Neurol Neurosurg Psychiatry 2014;85:660-667.

3. Anderson CS, Huang Y, Wang JG, et al. Intensive blood pressure reduction in acute cerebral haemorrhage trial (INTERACT): a randomised pilot trial. Lancet Neurol 2008;7:391-399.

4. Feigin VL, Forouzanfar MH, Krishnamurthi R, et al. Global and regional burden of stroke during 1990-2010: findings from the Global Burden of Disease Study 2010. Lancet 2014;383:245-254.

5. Richard F Keep Y, Guohua Xi. Intracerebral haemorrhage: mechanisms of injury and therapeutic targets. Lancet Neurol 2012;11: 720-731.

6. Kaya RA, Turkmenoglu O, Ziyal IM, Dalkilic T, Sahin Y, Aydin Y. The effects on prognosis of surgical treatment of hypertensive putaminal hematomas through transsylvian transinsular approach. Surg Neurol 2003;59:176-183; discussion 83.

7. Li Q, Yang CH, Xu JG, Li H, You C. Surgical treatment for large spontaneous basal ganglia hemorrhage: retrospective analysis of 253 cases. Br J Neurosurg 2013;27:617-621.

8. Background: National Health Insurance Research Database. Center for Biomedical Resources of NHRI. (Accessed 23 January 2017, at http://nhird.nhri.org.tw/en/.) 
9. Hsiao FY YC, Huang YT, Huang WF. Using Taiwan's National Health Insurance Research Databases for Pharmacoepidemiology Research. J Food Drug Anal 2007;15:99-108.

10. Huang WF, Hsiao FY, Wen YW, Tsai YW. Cardiovascular events associated with the use of four nonselective NSAIDs (etodolac, nabumetone, ibuprofen, or naproxen) versus a cyclooxygenase-2 inhibitor (celecoxib): a population-based analysis in Taiwanese adults. Clin Ther 2006;28:1827-1836.

11. Yang SY, Kao Yang YH, Chong MY, Yang YH, Chang WH, Lai CS. Risk of extrapyramidal syndrome in schizophrenic patients treated with antipsychotics: a population-based study. Clin Pharmacol Ther 2007;81:586-594.

12. Suzanne L. West MER, Charles Poole. Validity of Pharmacoepidemiologic Drug and Diagnosis Data: John Wiley \& Sons, Ltd. Hoboken, 2013.

13. Fisher ES, Whaley FS, Krushat WM, et al. The accuracy of Medicare's hospital claims data: progress has been made, but problems remain. Am J Public Health 1992;82:243-248.

14. Cheng CL, Kao YH, Lin SJ, Lee CH, Lai ML. Validation of the National Health Insurance Research Database with ischemic stroke cases in Taiwan. Pharmacoepidemiol Drug Saf 2011;20:236-242.

15. Broderick J, Connolly S, Feldmann E, et al. Guidelines for the management of spontaneous intracerebral hemorrhage in adults: 2007 update: a guideline from the American Heart Association/American Stroke Association Stroke Council, High Blood Pressure Research Council, and the Quality of Care and Outcomes in Research Interdisciplinary Working Group. Circulation 2007;116:e391-e413.

16. Mendelow AD, Gregson BA, Fernandes HM, et al. Early surgery versus initial conservative treatment in patients with spontaneous supratentorial intracerebral haematomas in the International Surgical Trial in Intracerebral Haemorrhage (STICH): a randomised trial. Lancet 2005;365:387-397.

17. Mendelow AD, Gregson BA, Rowan EN, et al. Early surgery versus initial conservative treatment in patients with spontaneous supratentorial lobar intracerebral haematomas (STICH II): a randomised trial. Lancet 2013;382:397-408.

18. Hanley DF, Thompson RE, Muschelli J, et al. Safety and efficacy of minimally invasive surgery plus alteplase in intracerebral haemorrhage evacuation (MISTIE): a randomised, controlled, open-label, phase 2 trial. Lancet Neurol 2016;15:1228-1237.

19. Mould WA, Carhuapoma JR, Muschelli J, et al. Minimally invasive surgery plus recombinant tissue-type plasminogen activator for intracerebral hemorrhage evacuation decreases perihematomal edema. Stroke 2013;44:627-634.

20. Spiotta AM, Fiorella D, Vargas J, et al. Initial multicenter technical experience with the Apollo device for minimally invasive intracerebral hematoma evacuation. Neurosurgery 2015;11 Suppl 2:243251; discussion 51.

21. Przybylowski CJ, Ding D, Starke RM, Webster Crowley R, Liu $\mathrm{KC}$. Endoport-assisted surgery for the management of spontaneous intracerebral hemorrhage. J Clin Neurosci 2015;22: $1727-1732$.

22. Egashira Y, Hua Y, Keep RF, Xi G. Intercellular cross-talk in intracerebral hemorrhage. Brain Res 2015;1623:97-109.

23. Aronowski J, Zhao X. Molecular pathophysiology of cerebral hemorrhage: secondary brain injury. Stroke 2011;42:1781-1786.

24. Arboix A, Comes E, Garcia-Eroles L, et al. Site of bleeding and early outcome in primary intracerebral hemorrhage. Acta Neurol Scand 2002;105:282-288.

25. Broderick JP. The STICH trial: what does it tell us and where do we go from here? Stroke 2005;36:1619-1620.

26. Ye Z, Ai X, Hu X, Fang F, You C. Comparison of neuroendoscopic surgery and craniotomy for supratentorial hypertensive intracerebral hemorrhage: A meta-analysis. Medicine 2017;96:e7876.

27. Prabhakaran $\mathrm{S}$, Naidech $\mathrm{AM}$. Ischemic brain injury after intracerebral hemorrhage: a critical review. Stroke 2012;43:2258-2263. 